\title{
MitoTEMPO Prevents Oxalate Induced Injury in NRK-52E Cells via Inhibiting Mitochondrial Dysfunction and Modulating Oxidative Stress
}

\author{
Jiaqiao Zhang, ${ }^{1}$ Qing Wang, ${ }^{1}$ Chuou Xu, ${ }^{2}$ Yuchao Lu, ${ }^{1}$ Henglong Hu, ${ }^{1}$ Baolong Qin, ${ }^{1}$ \\ Yufeng Wang, ${ }^{1}$ Deng He, ${ }^{3}$ Cong Li, ${ }^{1}$ Xiao Yu, ${ }^{1}$ Shaogang Wang, ${ }^{1}$ and Jihong Liu ${ }^{1}$ \\ ${ }^{1}$ Department and Institute of Urology, Tongji Hospital, Tongji Medical College, Huazhong University of Science and Technology, \\ Wuhan, Hubei, China \\ ${ }^{2}$ Department of Radiology, Tongji Hospital, Tongji Medical College, Huazhong University of Science and Technology, \\ Wuhan, Hubei, China \\ ${ }^{3}$ Department of Gynecology and Obstetrics, Tongji Hospital, Tongji Medical College, Huazhong University of Science and Technology, \\ Wuhan, Hubei, China
}

Correspondence should be addressed to Shaogang Wang; sgwangtjm@163.com

Received 23 October 2016; Revised 2 December 2016; Accepted 15 December 2016; Published 2 January 2017

Academic Editor: Mohamed M. Abdel-Daim

Copyright (C) 2017 Jiaqiao Zhang et al. This is an open access article distributed under the Creative Commons Attribution License, which permits unrestricted use, distribution, and reproduction in any medium, provided the original work is properly cited.

As one of the major risks for urolithiasis, hyperoxaluria can be caused by genetic defect or dietary intake. And high oxalate induced renal epithelial cells injury is related to oxidative stress and mitochondrial dysfunction. Here, we investigated whether MitoTEMPO, a mitochondria-targeted antioxidant, could protect against oxalate mediated injury in NRK-52E cells via inhibiting mitochondrial dysfunction and modulating oxidative stress. MitoSOX Red was used to determine mitochondrial ROS (mtROS) production. Mitochondrial membrane potential $(\Delta \psi \mathrm{m})$ and quantification of ATP synthesis were measured to evaluate mitochondrial function. The protein expression of Nox4, Nox2, and p22 was also detected to explore the effect of oxalate and MitoTEMPO on NADPH oxidase. Our results revealed that pretreatment with MitoTEMPO significantly inhibited oxalate induced lactate dehydrogenase (LDH) and malondialdehyde (MDA) release and decreased oxalate induced mtROS generation. Further, MitoTEMPO pretreatment restored disruption of $\Delta \psi \mathrm{m}$ and decreased ATP synthesis mediated by oxalate. In addition, MitoTEMPO altered the protein expression of Nox 4 and p22 and decreased the protein expression of IL-6 and osteopontin (OPN) induced by oxalate. We concluded that MitoTEMPO may be a new candidate to protect against oxalate induced kidney injury as well as urolithiasis.

\section{Introduction}

Hyperoxaluria is considered as one of the primary risk factors for urolithiasis [1]. High oxalate can cause renal tubular cells injury, and this effect is related to reactive oxygen species (ROS) production and imbalance of oxidation-reduction [2]. Mitochondria and NADPH oxidase are the major sources of ROS generation in renal tubular cells, and mitochondrial dysfunction was regarded as a key event in oxalate mediated renal cell injury [3-7].

Various antioxidant treatments including NADPH oxidase inhibitors had been applied to prevent renal cell injury and/or renal crystal formation [8-13]. However, limited research focused on preventing oxalate mediated renal cell toxicity via targeting mitochondria. Further, mitochondriatargeted antioxidant may scavenge mitochondrial ROS (mtROS) without affecting physiological ROS production outside the mitochondria [14].

Targeted therapies for mtROS species have not been reported in oxalate mediated injury in renal tubular cells. MitoTEMPO, a mitochondria-targeted antioxidant can specifically accumulate in the mitochondrial matrix 1000fold due to its positive charge [15]. We hypothesized that MitoTEMPO prevents oxalate induced injury in renal tubular 
cell via inhibiting mitochondrial dysfunction and modulating oxidative stress. We hoped to find new and more specific drugs to treat oxalate induced kidney injury as well as urolithiasis.

\section{Materials and Methods}

2.1. Reagents. MitoTEMPO was purchased from SigmaAldrich (St. Louis, MO, USA) and was dissolved in deionized water according to the molecular weight. Cell counting kit 8 (CCK-8) was purchased from Signalway Antibody LLC (Maryland, USA). LDH cytotoxicity assay kit, 2,7dichlorodihydrofluorescein diacetate (DCF-DA), JC-1 dye and MDA assay kit, and ATP assay kit were purchased from Beyotime Institute of Biotechnology (Jiangsu, China). MitoSOX Red was purchased from Molecular ProbesInvitrogen (Carlsbad, CA, USA). OPN and Nox4 antibodies were purchased from Abcam (Cambridge, MA, USA); IL6 antibody was purchased from Biorbyt (Cambridge, United Kingdom); p22 antibody was purchased from Santa Cruz Biotechnology (Santa Cruz, CA, USA); Nox2 and $\beta$ actin antibodies were purchased from Proteintech (Wuhan, China).

2.2. Cell Culture. The NRK-52E (normal rat proximal tubular epithelial cell line) was obtained from the Type Culture Collection of the Chinese Academy of Sciences (Shanghai, China). Cells were maintained in Dulbecco's Modified Eagle's Medium (DMEM) (Hyclone; USA) supplemented with 10\% fetal bovine serum (Gibco; Grand Island, NY, USA) at $37^{\circ} \mathrm{C}$ under a humidified $5 \% \mathrm{CO}_{2}$ atmosphere. Under these conditions, the cells achieved $90 \%$ to confluence. They were washed with serum and sodium pyruvate-free DMEM media.

2.3. Detection of Cell Viability (CCK-8). The NRK-52E cells were seeded at the concentration of $2 \times 10^{3} /$ well in 96 -well plates and incubated overnight. Following pretreatment with or without MitoTEMPO $(10 \mu \mathrm{M})$ for 1 hour, the cells were treated with or without oxalate $(700 \mu \mathrm{mol} / \mathrm{l})$ for 24 or 48 hours at $37^{\circ} \mathrm{C}$. After treatment, $100 \mu \mathrm{l}$ DMEM media with $10 \%$ CCK-8 were added to replace previous media in each well and incubated for another 3 hours. The absorbance at $450 \mathrm{~nm}$ was detected. The viability of the cells was recorded as the percentage relative to untreated controls.

2.4. Lactate Dehydrogenase ( $L D H)$ Assay. The release of $\mathrm{LDH}$ into media was measured to detect the injury effect on NRK52E cells. Cells were seeded in 96-well plates and cultured overnight. Before treatment, they were replaced with serum and sodium pyruvate-free DMEM media. After treatment for various periods, supernatants were collected and detected according to related instruction manual. The $\mathrm{LDH}$ release activity was presented as the fold increase over the control group.

2.5. Detection of Total Intracellular ROS by Flow Cytometry. Intracellular ROS production was detected using the ROS assay kit as the fluorescence of 2,7-dichlorofluorescein diacetate (DCF-DA) significantly increases after oxidation. Cells were seeded in a 6-well plate and stimulated by oxalate with or without 1 hour of preincubation with MitoTEMPO. After $3 \mathrm{~h}$, cells were treated with DCF-DA $(10 \mu \mathrm{M})$ for $30 \mathrm{~min}$. Then, the cells were collected and resuspended in $200 \mu \mathrm{l}$ of PBS buffer. Then intracellular ROS levels were performed by flow cytometry (BD Biosciences) with extinction and emission at 485 and $538 \mathrm{~nm}$. All determinations were performed in triplicate.

2.6. Determination of mtROS Levels by Flow Cytometry. MitoSOX Red mitochondrial superoxide indicator was used to determine mtROS production. The cells were treated as the same when detecting intracellular ROS. After treatment, cells were loaded with $5 \mu \mathrm{M}$ MitoSOX Red reagent for $10 \mathrm{~min}$ at $37^{\circ} \mathrm{C}$. Then the cells were collected, washed, and resuspended in $200 \mu \mathrm{l}$ Hanks' balanced salt solution. Flow cytometric analyses were performed with extinction and emission at 510 and $580 \mathrm{~nm}$ to determine mtROS. All determinations were performed in triplicate.

2.7. Assay of Lipid Peroxidation (MDA). Lipid peroxidation was measured as malondialdehyde (MDA) content. MDA was determined according to the instructions. The levels of MDA were expressed as percentage relative to the controls.

2.8. Detection of Mitochondrial Membrane Potential (MMP) by Fluorescence Microscopy. MMP assay kit (JC-1) was used to detect MMP. Cells were cultured in six-well plates. After stimulation by oxalate with or without 1 hour of preincubation with MitoTEMPO for 3 hours, cells were incubated with dyeing working solution for 20 minutes at $37^{\circ} \mathrm{C}$, protected from light. JC-1 selectively entering mitochondria exists as a monomer with green fluorescence $(E m 530 \mathrm{~nm}$ ) at relatively low membrane potential and aggregates with red fluorescence $(E m 590 \mathrm{~nm})$ at high membrane potential. Then cells were observed by the fluorescence microscope (Olympus IX73, Japan).

2.9. Determination of Cellular ATP. Cellular ATP levels were determined using a commercial ATP assay kit based on the luciferin-luciferase reaction. After treatments, NRK-52E cells were lysed and centrifuged at $12,000 \mathrm{~g}$ for $5 \mathrm{~min}$. Then, $20 \mu \mathrm{L}$ supernatants were mixed with $100 \mu \mathrm{L}$ detection working solution in a black 96-well plate. Then, the chemiluminescence was measured. The level of ATP was expressed as percentage relative to the controls.

2.10. Western Blotting Analysis. After treatment, the cells were washed and lysed with RIPA buffer that contained protease inhibitor. Same amounts of proteins in each sample were analyzed on $10 \%$ SDS-PAGE. After being transferred to PVDF membranes, the membranes were blocked with $3 \% \mathrm{BSA}$ for $2 \mathrm{~h}$ and then the membranes were incubated with primary antibody (1:500 for rabbit polyclonal antiNox2; 1:1,000 for rabbit monoclonal anti-NADPH oxidase 4; 1:250 for mouse monoclonal anti-P22; 1:1000 for rabbit monoclonal anti-OPN; 1:200 for rabbit polyclonal anti-IL6; $1: 500$ for mouse monoclonal $\beta$-actin) at $4^{\circ} \mathrm{C}$ overnight; the signals of the membranes were visualized using enhanced 


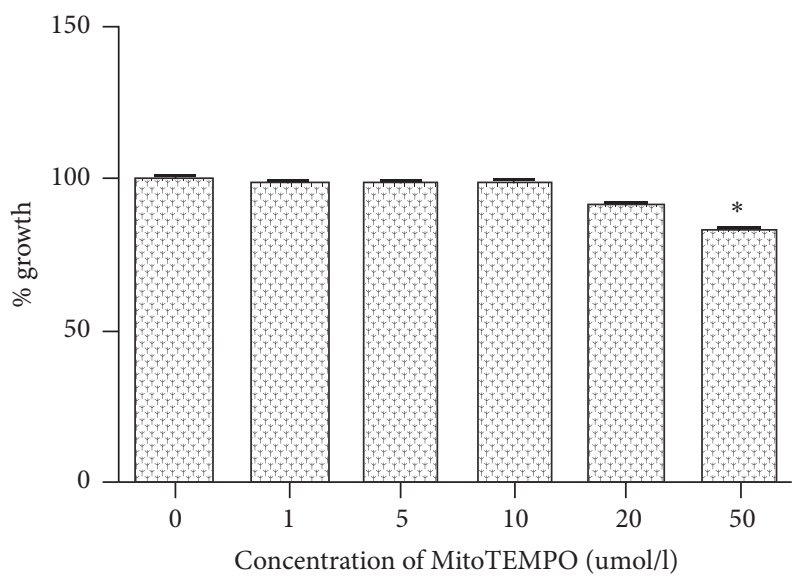

(a)

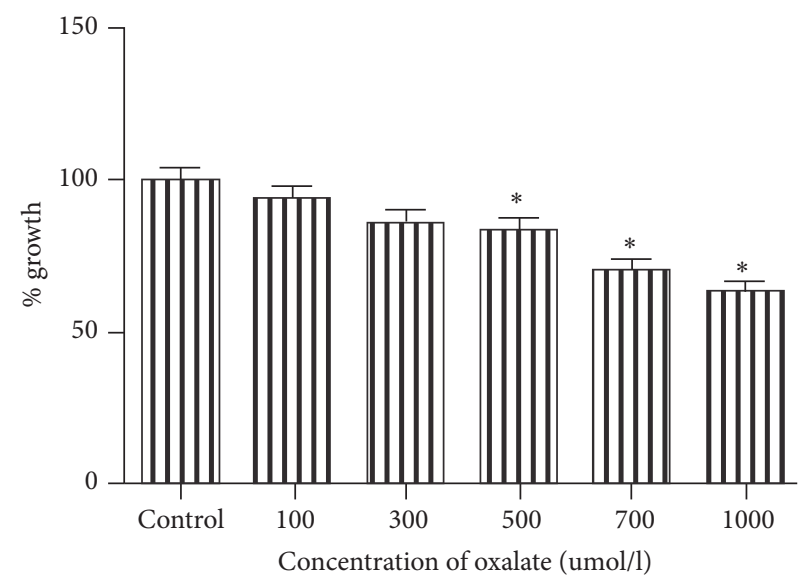

(b)

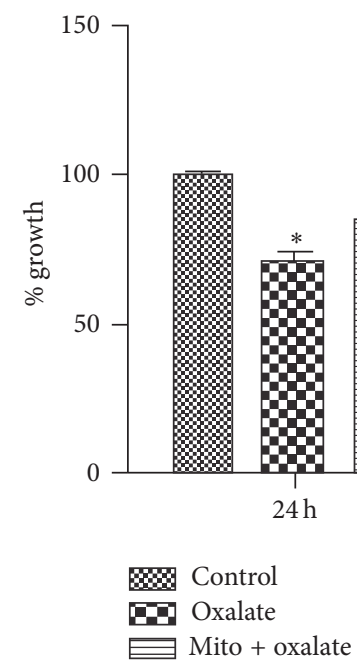

(c)

FIGURE 1: Effects of oxalate and MitoTEMPO on NRK-52E cell viability (a, b, c). The viability of NRK-52E cells was assessed by performing a CCK-8 assay. (a) Cells were treated with MitoTEMPO in different concentrations $(0-50 \mu \mathrm{M})$. (b) Cells were treated with potassium oxalate in different concentrations $(0-1000 \mu \mathrm{M})$. (c) MitoTEMPO $(10 \mu \mathrm{M}$, pretreatment for 1 hour) increased NRK-52E cell viability in the presence of oxalate $(700 \mu \mathrm{M})$ at 24 and 48 hours. ${ }^{*} P<0.05$ versus control, ${ }^{\#} P<0.05$ versus oxalate treated group ( ${ }^{*}$ Mito: MitoTEMPO).

chemiluminescence (ECL) after being incubated with secondary antibody (anti-rabbit or anti-mouse IgG) for $1 \mathrm{~h}$ at room temperature. Anti- $\beta$-actin was used to normalize the protein expression.

2.11. Statistical Analysis. Results were expressed as the mean \pm SEM. One-way ANOVA was used to determine statistically significant differences in these experiments by GraphPad Prism 5 software.

\section{Results}

3.1. Effects of Oxalate and MitoTEMPO on NRK-52E Cell Viability. No significant toxicity of MitoTEMPO (1 to $20 \mu \mathrm{M})$ was found for NRK-52E cells for 24 hours (Figure 1(a)). The oxalate treatment (for 24 hours) decreased the viability of cells in a dose-dependent manner (Figure 1(b)). According to that, oxalate with concentration of $700 \mu \mathrm{M}$ was selected for the following experiments. After pretreatment with MitoTEMPO $(10 \mu \mathrm{M})$ for 1 hour, cells were exposed to oxalate $(700 \mu \mathrm{M})$ for 24 hours to check cell viability. And we found that treatment with MitoTEMPO could significantly increase the cells viability (Figure 1(c)).

3.2. MitoTEMPO Attenuates Oxalate Mediated Cell Injury. To further test the protection of MitoTEMPO against oxalate induced cell injury, the LDH release activity was detected. Exposure of the cells to oxalate $(700 \mu \mathrm{M})$ resulted in the release of LDH, significantly more than in the controls. In contrast, MitoTEMPO effectively reduced LDH leakage caused by oxalate at different periods ( 3 to 24 hours) (Figure 2(a)).

3.3. MitoTEMPO Reduces Lipid Peroxidation Injury in NRK$52 E$ Cells Exposed to Oxalate. MDA levels were examined as 


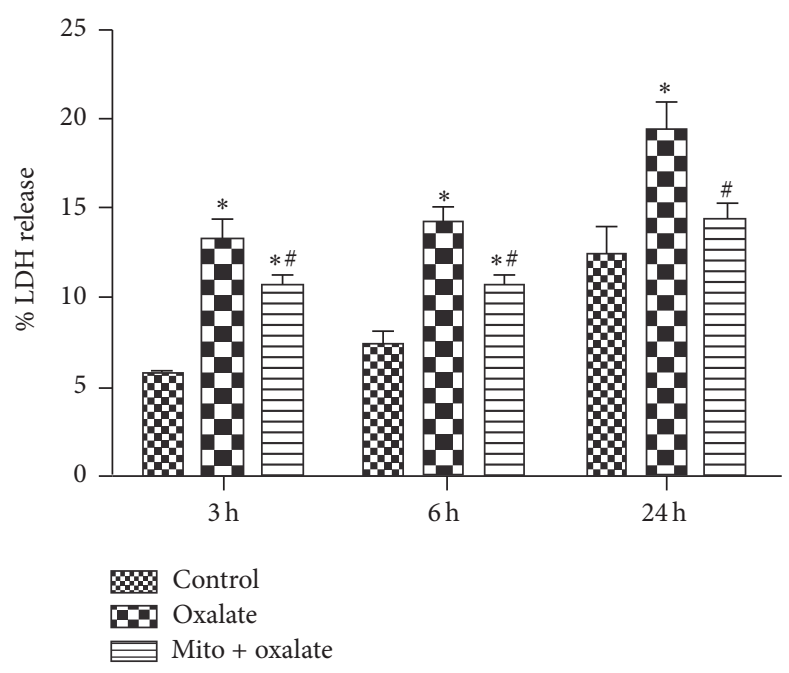

(a)
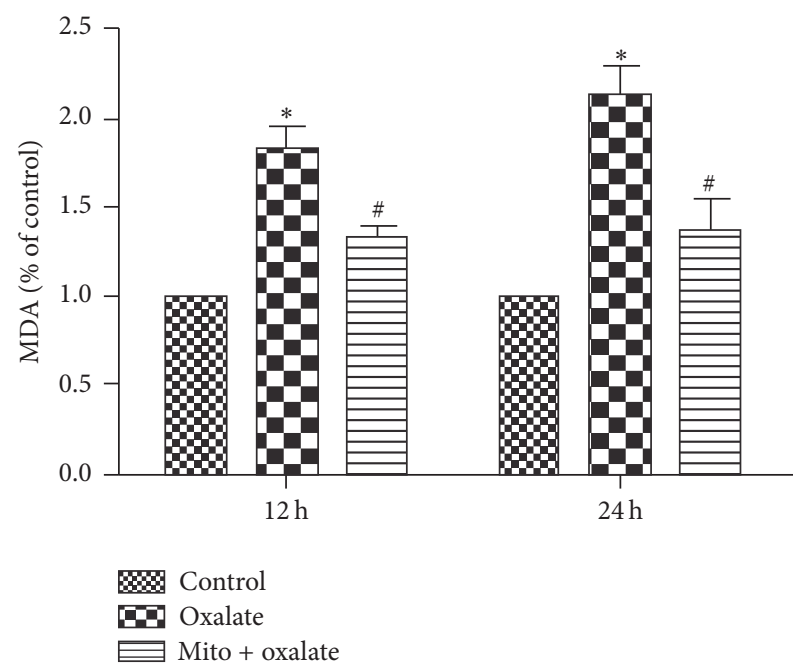

(b)

Figure 2: Mito attenuates oxalate induced cell injury and lipid peroxidation (a, b). (a) Mito decreased oxalate induced LDH release at 3, 6 , and 24 hours. (b) Lipid peroxidation was assessed by detecting MDA level in the supernatants of NRK cell lysates. Pretreatment with Mito (1 hour) obviously attenuated MDA generation compared with oxalate induced group. ${ }^{*} P<0.05$ versus control; ${ }^{\#} P<0.05$ versus oxalate treated group ( ${ }^{*}$ Mito: MitoTEMPO).

the marker of lipid peroxidation injury and oxidative stress. Treatment with oxalate significantly increased cellular MDA content, and pretreatment with MitoTEMPO significantly decreased the cellular MDA levels compared with oxalate induced cells (Figure 2(b)).

\subsection{MitoTEMPO Attenuates Oxalate Induced Mitochondrial} ROS (mtROS) but not Intracellular ROS Generation. The DCF-DA fluorescence was measured to determine total intracellular ROS level and the intensity of MitoSOX Red fluorescence to determine the mtROS level. Pretreatment with MitoTEMPO significantly decreased the mtROS generation compared with oxalate induced cells; however it had no obvious impact on intracellular ROS generation (Figure 3).

3.5. MitoTEMPO Ameliorates Oxalate Induced Disruption of Mitochondrial Membrane Potential. Relative to the control group, oxalate exposure produced an obvious reduction in the initial $\Delta \psi_{\mathrm{m}}$, as estimated by the decreased JC-1 fluorescence at $596 \mathrm{~nm}$ (red) and concomitant increased fluorescence at $534 \mathrm{~nm}$ (green). With MitoTEMPO pretreatment for $1 \mathrm{~h}$, an obvious restoration of MMP was observed (Figure 4). The results indicate that oxalate induced mitochondrial dysfunction, and MitoTEMPO could inhibit this effect.

3.6. MitoTEMPO Increases ATP Levels in the NRK-52E Cells Exposed to Oxalate. Decreased MMP is related to reduction of ATP synthesis. Our results showed that oxalate could induce ATP deficiency in the NRK-52E cells, and MitoTEMPO treatment restored this effect (Figure 5).

3.7. MitoTEMPO Selectively Modulated the Protein Expression of NADPH Oxidase Subunits. It was reported that oxalate exposure could regulate mRNA expression of NADPH oxidase subunits in a human renal epithelial-derived cell line [5]. In this research, we detected the effect of oxalate and MitoTEMPO on the protein expression of NADPH oxidase subunits. Oxalate treatment decreased the protein expression of Nox4, elevated the protein expression of p22 significantly, and did not change the protein expression of Nox2. MitoTEMPO pretreatment reversed the change of protein expression of Nox4 and p22 without affecting the protein expression of Nox2 (Figure 6).

3.8. MitoTEMPO Reduced the Expression of OPN and IL-6 in NRK-52E Cells Exposed to Oxalate. Oxalate significantly increased the protein expression of OPN and IL-6. Furthermore, the increased expression of OPN and IL-6 was significantly attenuated when cells were pretreated with MitoTEMPO (Figure 7).

\section{Discussion}

Numerous studies have indicated that ROS and oxidative stress were involved in the stone formation as well as oxalate induced renal epithelial cells injury. As two important sources of ROS production, both mitochondria and NADPH oxidases were involved in oxalate induced renal epithelial cells injury $[3,6,13,16,17]$.

However, ROS generation is not always detrimental, as it is involved in both physiological and pathological processes. Many intervention trials with antioxidants supposed to prevent oxidative stress and improve disease outcomes have been mostly invalid or harmful. An important reason for these failures may be attributed to inappropriate inhibition of physiological ROS [18]. Therefore, theoretically we should intervene 

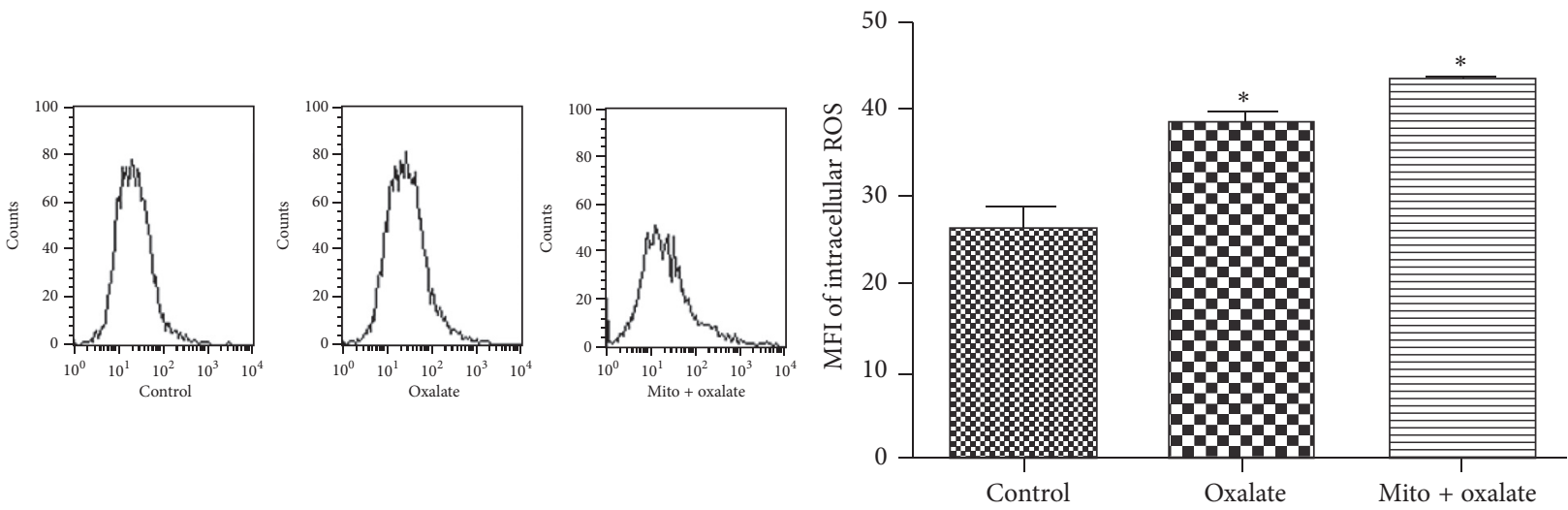

(a)
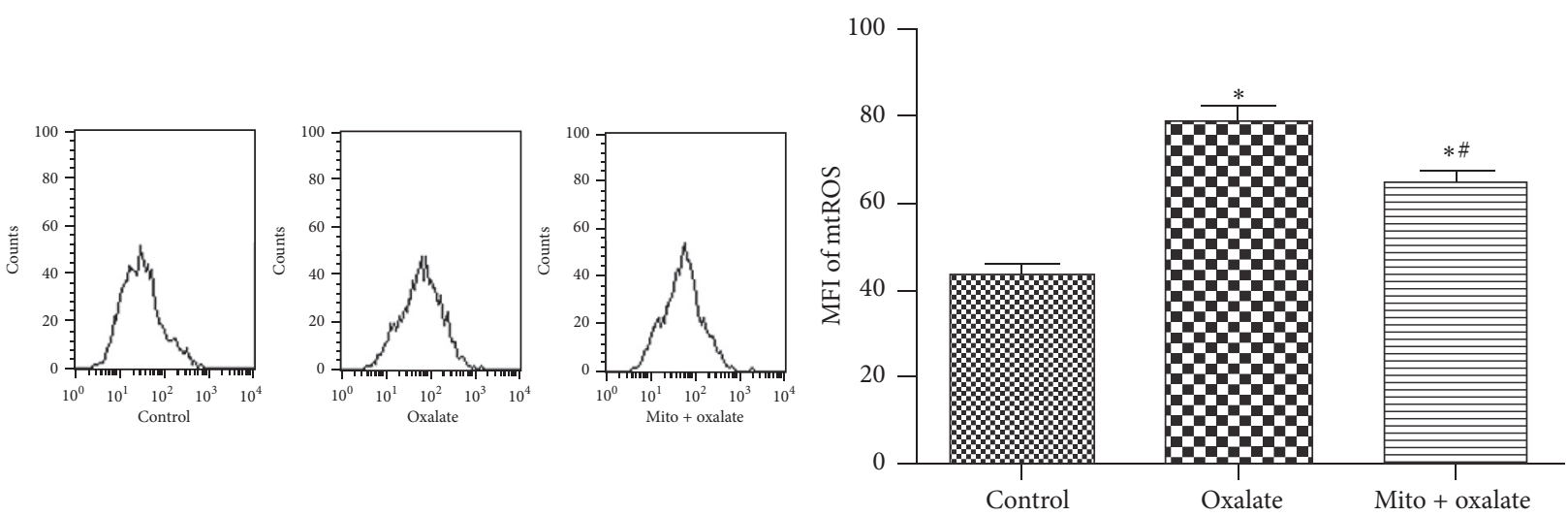

(b)

FIGURE 3: Effect of MitoTEMPO on oxalate induced intracellular ROS and mtROS generation (a, b). NRK-52E cells were stimulated with oxalate $(700 \mu \mathrm{M})$ for 3 hours with or without preincubation with MitoTEMPO for 1 hour. (a) The intracellular ROS levels were indicated by the fluorescence intensity of DCF analyzed by flow cytometry. (b) The mtROS levels were indicated by the fluorescence intensity of MitoSOX Red analyzed by flow cytometry. Mean \pm SEM of three independent experiments. ${ }^{*} P<0.05$ versus control; ${ }^{*} P<0.05$ versus oxalate treated group ( ${ }^{*}$ Mito: MitoTEMPO).
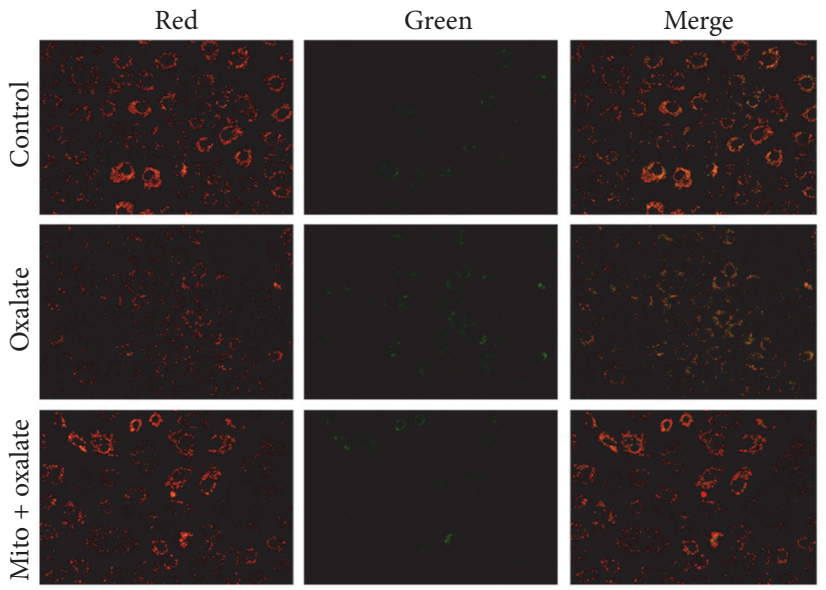

FIGURE 4: Mito ameliorates oxalate induced disruption of mitochondrial membrane potential. NRK-52E cells were stimulated with oxalate $(700 \mu \mathrm{M})$ for 1 hour with or without preincubation with MitoTEMPO for 1 hour. Mitochondrial membrane potential was observed using a mitochondrial potential-sensitive dye, JC-1. Cells in control group showed bright red fluorescence. Oxalate treatment attenuated red fluorescence compared with control, and pretreatment with MitoTEMPO reversed these changes ( ${ }^{*}$ Mito: MitoTEMPO). 


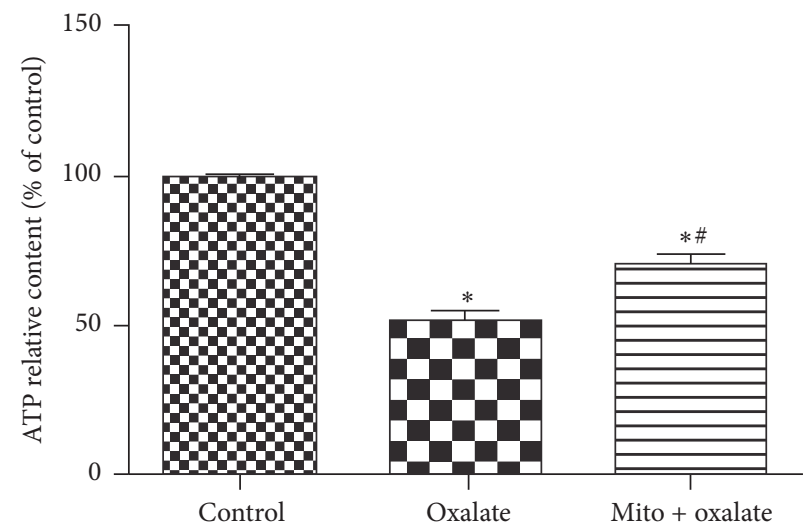

FIGURE 5: Mito increases ATP levels in the oxalate induced cultured NRK52E cells. NRK-52E cells were stimulated with oxalate (700 $\mu \mathrm{M})$ for 3 hours with or without preincubation with MitoTEMPO for 1 hour. Oxalate treatment decreased ATP levels significantly. ATP levels in MitoTEMPO pretreatment group significantly increased compared with oxalate treatment group and still was lower compared with control group. ${ }^{*} P<0.05$ versus control; ${ }^{\#} P<0.05$ versus oxalate treated group $\left({ }^{*}\right.$ Mito: MitoTEMPO $)$.
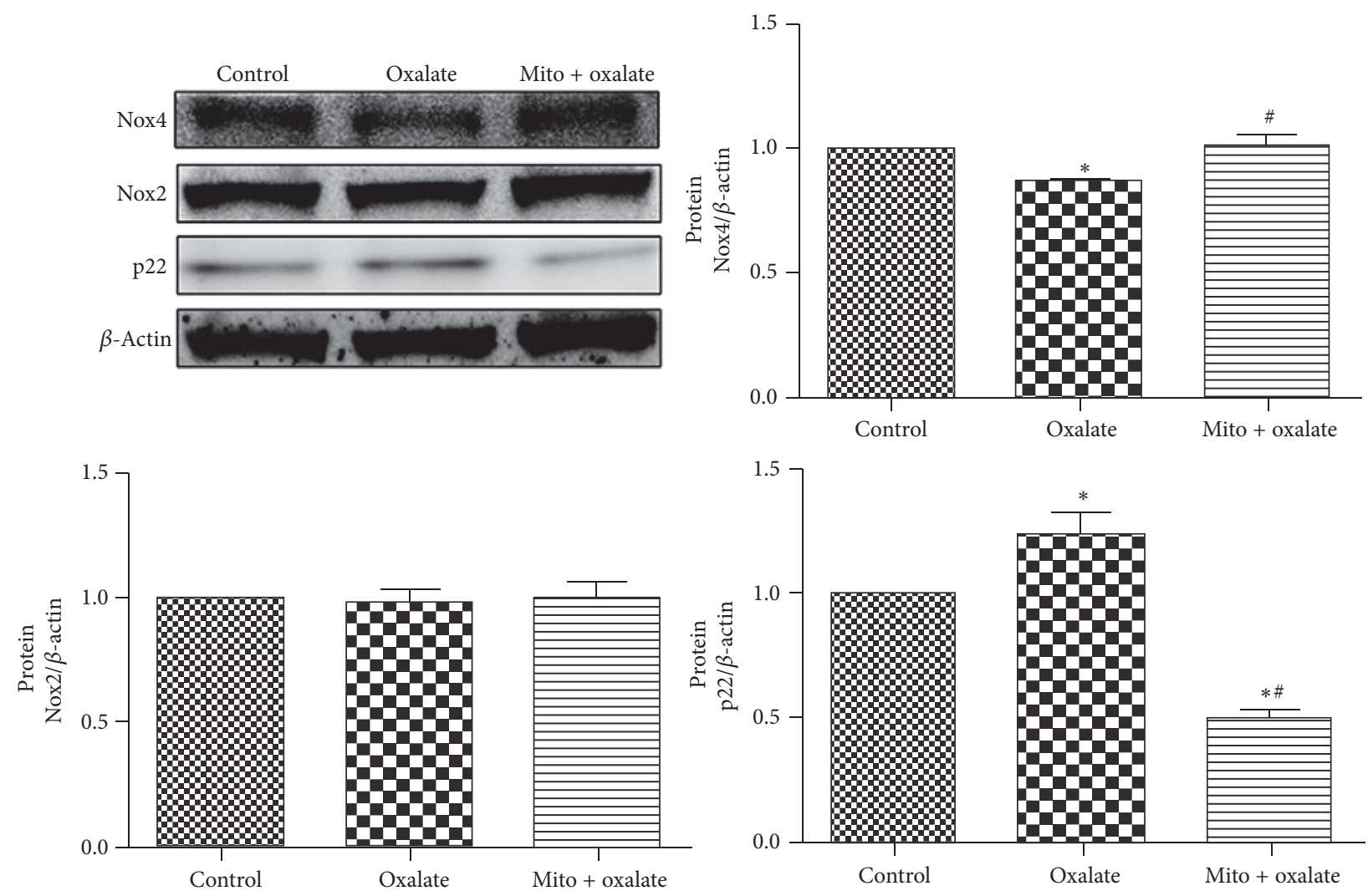

FIGURE 6: MitoTEMPO selectively modulated the protein expression of NADPH oxidase subunits in NRK-52E cells exposed to oxalate. NRK-52E cells were stimulated with oxalate $(700 \mu \mathrm{M})$ for 24 hours with or without preincubation with MitoTEMPO for 1 hour. Oxalate treatment decreased the protein expression of Nox 4 and elevated the protein expression of p 22 significantly compared with the control group. MitoTEMPO pretreatment elevated the protein expression of Nox4 and decreased the protein expression of p22. The protein expression of Nox2 was not changed after oxalate stimulation with or without MitoTEMPO pretreatment. ${ }^{*} P<0.05$ versus control; ${ }^{\#} P<0.05$ versus oxalate treated group $\left({ }^{*}\right.$ Mito: MitoTEMPO). 

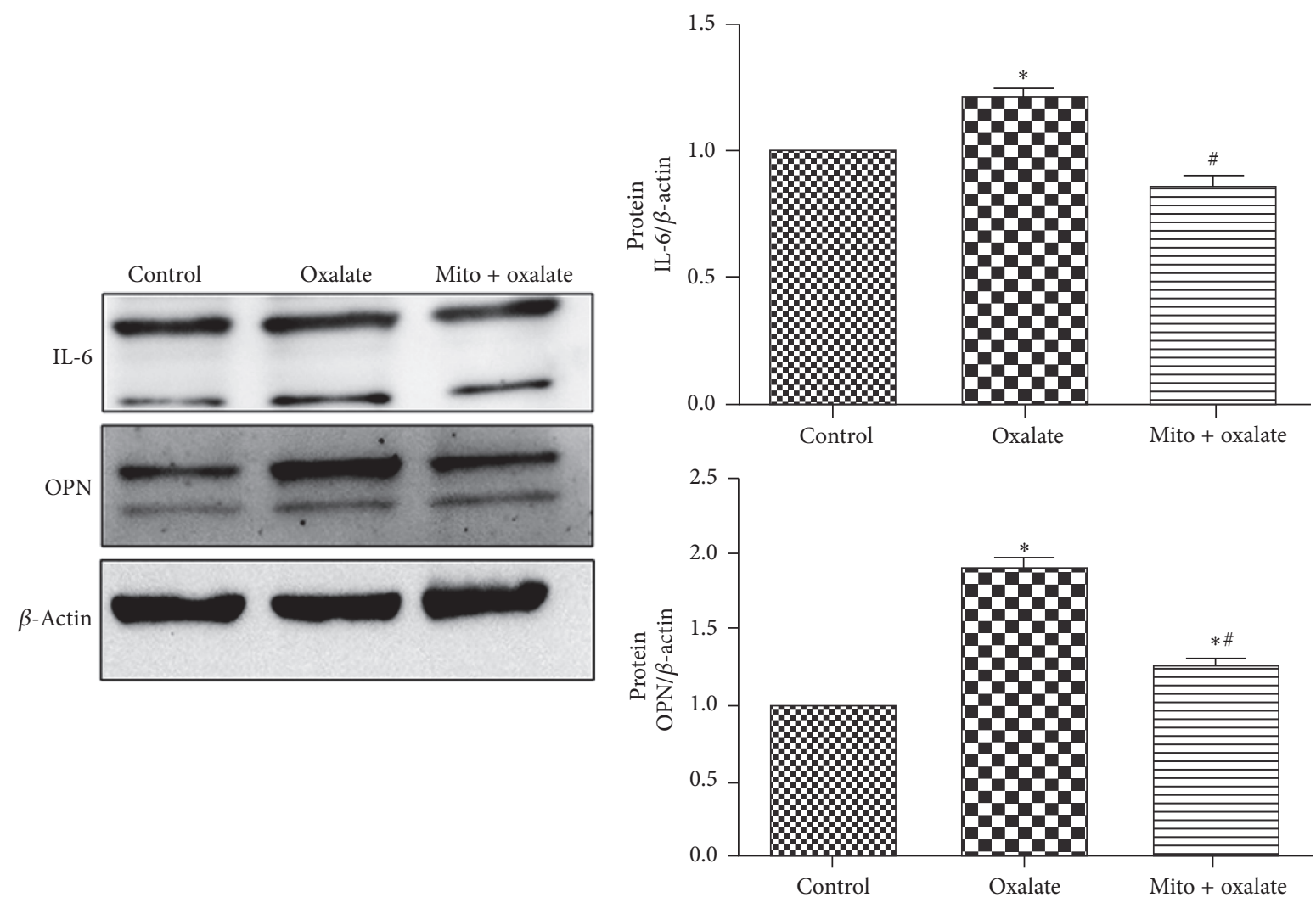

FIGURE 7: MitoTEMPO reduced the expression of stone modulators of IL-6 and OPN in NRK-52E cells exposed to oxalate. NRK-52E cells were stimulated with oxalate $(700 \mu \mathrm{M})$ for 24 hours with or without preincubation with MitoTEMPO for 1 hour $\left({ }^{*}\right.$ Mito: MitoTEMPO).

by targeting pathological ROS while making physiologically relevant ROS unaffected. Interestingly, it was reported that mtROS could directly contribute to inflammatory cytokine production and we suggested that mtROS may be a type of pathological ROS $[19,20]$. Based on these evidences, we propose a hypothesis that MitoTEMPO, a mitochondriatargeted antioxidant, would exert a protective effect against oxalate induced renal epithelial cells injury via specifically acting on mitochondria and modulating ROS generation.

In our study, MitoTEMPO significantly increased the cells viability following exposure to oxalate. Also pretreatment with MitoTEMPO obviously decreased NRK-52E cells injury assessed by LDH release. The marker of oxidative stress, MDA level, was also decreased in MitoTEMPO-treated group. We also demonstrated that MitoTEMPO increased the cells viability via inhibiting mitochondrial ROS generation and restoration of mitochondrial membrane potential as well as promoting ATP synthesis.

Interestingly, our research found that MitoTEMPO attenuated mtROS generation caused by oxalate stimulation but not intracellular ROS generation. This phenomenon may be because MitoTEMPO can selectively accumulate in the mitochondria. Of course, there may be interaction between mitochondria and NADPH oxidase. Research indicated that oxidative stress provokes a positive-feedback loop between the two, although exact mechanism is undefined $[15,21]$. As previous studies have indicated oxalate could activate the NADPH oxidase and upregulate the expression of some NADPH oxidase subunits $[5,17]$, we wondered if NADPH oxidase subunits could be regulated by MitoTEMPO treatment. And we observed that the protein expressions of Nox 4 and p22 were also changed after oxalate and oxalate and MitoTEMPO combination treatment. This result also indicated mtROS may regulate NADPH oxidase activity via regulating the protein expression of NADPH oxidase subunits although we did not detect the activity of the NADPH oxidase.

The family of NADPH oxidases consists of seven isoforms (Nox1-5, Duoxl, and Duox2). Of the seven isoforms, Nox4 and Nox 2 are the two major components in the kidney, and activation of both Nox 4 and Nox 2 depends on p22 [22]. In contrast to NADPH oxidase Nox2, Nox4 produces hydrogen peroxide rather than superoxide [23]. Based on these evidences, we speculate that MitoTEMPO may play a protective role in oxalate mediated cell injury through modulating not only "dose" but also "type" of ROS generation.

As one of the proinflammatory cytokines, IL-6 may be involved in pathogenesis of urolithiasis. It was reported that patients of urolithiasis were associated with elevated level of IL-6 [24]. Study also indicated that oxalate could increase the expression of IL-6 in a time- and concentration-dependent manner [25]. As publications have discovered that mtROS acted as signaling molecules to lead inflammatory cytokine production [26-28], we determine the effect of oxalate and 
MitoTEMPO on the expression of IL-6. And our results demonstrated that oxalate also upregulated the expression of IL- 6 in NRK-52E cells, and MitoTEMPO treatment could weaken this effect.

Osteopontin (OPN), secreted by renal tubular epithelial cells, is considered as an important macromolecular modulator in the development of urolithiasis. The OPN expression is upregulated in both hyperoxaluric rats and genetic hypercalciuric rats $[29,30]$. Studies also revealed that high concentration of calcium, oxalate, or calcium oxalate monohydrate (COM) crystals could stimulate OPN expression in renal tubular cells $[4,31,32]$. In our study, MitoTEMPO modulated ROS (including mitochondrial and intracellular) generation and OPN expression induced by oxalate. It is consistent with previous reports that OPN expression is regulated by ROS $[4,33]$.

There are some limitations in our study. Firstly, we just determined a part of NADPH oxidase subunits and did not include NADPH oxidase activity. Secondly, we did not detect specific type of intracellular ROS, for example, hydrogen peroxide or superoxide anion. At last, how MitoTEMPO affects downstream redox signaling such as p38, JNK, and ERK1/2 pathways is yet to be known.

In our study, MitoTEMPO may play a role to inhibit "harmful" ROS to confer protection against oxalate induced cell injury. Furthermore, MitoTEMPO decreased protein expression of IL-6 and OPN which are sensitive to redox regulation. To our knowledge, it is the first time to apply mitochondria-targeted antioxidant to inhibit oxalate induced cytotoxicity. Our present study implicates that MitoTEMPO may be an ideal candidate to control oxalate induced kidney injury as well as urolithiasis.

\section{Competing Interests}

The authors declare that no conflict of interests exists.

\section{Authors' Contributions}

Jihong Liu, Shaogang Wang, Xiao Yu, Cong Li, and Jiaqiao Zhang conceived and designed the study. Yuchao Lu, Deng He, Baolong Qin, Yufeng Wang, Qing Wang, Chuou Xu, and Jiaqiao Zhang performed the experiments. Henglong $\mathrm{Hu}$ and Jiaqiao Zhang analyzed the data. Jiaqiao Zhang wrote the manuscript. All authors read and approved the manuscript.

\section{Acknowledgments}

This work was supported by National Natural Science Foundation of China (81270787, 81570631).

\section{References}

[1] D. W. Kaufman, J. P. Kelly, G. C. Curhan et al., "Oxalobacter formigenes may reduce the risk of calcium oxalate kidney stones," Journal of the American Society of Nephrology, vol. 19, no. 6, pp. 1197-1203, 2008.

[2] H. Tsuji, W. Wang, J. Sunil et al., "Involvement of reninangiotensin-aldosterone system in calcium oxalate crystal induced activation of NADPH oxidase and renal cell injury," World Journal of Urology, vol. 34, no. 1, pp. 89-95, 2016.

[3] F. D. Khand, M. P. Gordge, W. G. Robertson, A. A. NoronhaDutra, and J. S. Hothersall, "Mitochondrial superoxide production during oxalate-mediated oxidative stress in renal epithelial cells," Free Radical Biology \& Medicine, vol. 32, no. 12, pp. 13391350, 2002.

[4] T. Umekawa, H. Tsuji, H. Uemura, and S. R. Khan, "Superoxide from NADPH oxidase as second messenger for the expression of osteopontin and monocyte chemoattractant protein-1 in renal epithelial cells exposed to calcium oxalate crystals," BJU International, vol. 104, no. 1, pp. 115-120, 2009.

[5] S. R. Khan, A. Khan, and K. J. Byer, "Temporal changes in the expression of mRNA of NADPH oxidase subunits in renal epithelial cells exposed to oxalate or calcium oxalate crystals," Nephrology Dialysis Transplantation, vol. 26, no. 6, pp. 1778$1785,2011$.

[6] S. Joshi, A. B. Peck, and S. R. Khan, "NADPH oxidase as a therapeutic target for oxalate induced injury in kidneys," Oxidative Medicine and Cellular Longevity, vol. 2013, Article ID 462361, 18 pages, 2013.

[7] L.-C. Cao, T. W. Honeyman, R. Cooney, L. Kennington, C. R. Scheid, and J. A. Jonassen, "Mitochondrial dysfunction is a primary event in renal cell oxalate toxicity," Kidney International, vol. 66, no. 5, pp. 1890-1900, 2004.

[8] H.-S. Huang, J. Chen, C.-F. Chen, and M.-C. Ma, "Vitamin E attenuates crystal formation in rat kidneys: roles of renal tubular cell death and crystallization inhibitors," Kidney International, vol. 70, no. 4, pp. 699-710, 2006.

[9] X. Li, J. Lu, P. Shang, J. Bao, and Z. Yue, "The selective NADPH oxidase inhibitor apocynin has potential prophylactic effects on melamine-related nephrolithiasis in vitro and in vivo," Molecular and Cellular Biochemistry, vol. 399, no. 1, pp. 167-178, 2015.

[10] M. Alex, M. V. S. Paul, M. Abhilash, V. V. Mathews, T. V. Anilkumar, and R. H. Nair, "Astaxanthin modulates osteopontin and transforming growth factor $\beta 1$ expression levels in a rat model of nephrolithiasis: a comparison with citrate administration," $B J U$ International, vol. 114, no. 3, pp. 458-466, 2014.

[11] S. Thamilselvan and M. Menon, "Vitamin E therapy prevents hyperoxaluria-induced calcium oxalate crystal deposition in the kidney by improving renal tissue antioxidant status," BJU International, vol. 96, no. 1, pp. 117-126, 2005.

[12] J. Zuo, A. Khan, P. A. Glenton, and S. R. Khan, "Effect of NADPH oxidase inhibition on the expression of kidney injury molecule and calcium oxalate crystal deposition in hydroxyL-proline-induced hyperoxaluria in the male Sprague-Dawley rats," Nephrology Dialysis Transplantation, vol. 26, no. 6, pp. 1785-1796, 2011.

[13] S. Joshi, B. T. Saylor, W. Wang, A. B. Peck, and S. R. Khan, "Apocynin-treatment reverses hyperoxaluria induced changes in NADPH oxidase system expression in rat kidneys: a transcriptional study," PLoS ONE, vol. 7, no. 10, Article ID e47738, 2012.

[14] L. C. A. Tábara, J. Poveda, C. Martin-Cleary, R. Selgas, A. Ortiz, and M. D. Sanchez-Niño, "Mitochondria-targeted therapies for acute kidney injury," Expert reviews in molecular medicine, vol. 16, article e13, 2014.

[15] S. Dikalov, "Cross talk between mitochondria and NADPH oxidases," Free Radical Biology \& Medicine, vol. 51, no. 7, pp. 1289-1301, 2011. 
[16] S. M. Farooq, N. B. Boppana, D. Asokan et al., "C-phycocyanin confers protection against oxalate-mediated oxidative stress and mitochondrial dysfunctions in MDCK cells," PLoS ONE, vol. 9, no. 4, Article ID e93056, 2014.

[17] A. Khan, K. Byer, and S. R. Khan, "Exposure of madin-darby canine kidney (MDCK) cells to oxalate and calcium oxalate crystals activates nicotinamide adenine dinucleotide phosphate (NADPH)-oxidase," Urology, vol. 83, no. 2, pp. 510.e1-510.e7, 2014.

[18] A. I. Casas, V. T.-V. Dao, A. Daiber et al., "Reactive oxygenrelated diseases: therapeutic targets and emerging clinical indications," Antioxidants \& Redox Signaling, vol. 23, no. 14, pp. 11711185, 2015.

[19] X. Li, P. Fang, J. Mai, E. T. Choi, H. Wang, and X.-F. Yang, "Targeting mitochondrial reactive oxygen species as novel therapy for inflammatory diseases and cancers," Journal of Hematology \& Oncology, vol. 6, article 19, 2013.

[20] E. Naik and V. M. Dixit, "Mitochondrial reactive oxygen species drive proinflammatory cytokine production," The Journal of Experimental Medicine, vol. 208, no. 3, pp. 417-420, 2011.

[21] D. B. Zorov, M. Juhaszova, and S. J. Sollott, "Mitochondrial reactive oxygen species (ROS) and ROS-induced ROS release," Physiological Reviews, vol. 94, no. 3, pp. 909-950, 2014.

[22] M. Sedeek, R. Nasrallah, R. M. Touyz, and R. L. Hébert, "NADPH oxidases, reactive oxygen species, and the kidney: friend and foe," Journal of the American Society of Nephrology, vol. 24, no. 10, pp. 1512-1518, 2013.

[23] I. Takac, K. Schröder, L. Zhang et al., "The E-loop is involved in hydrogen peroxide formation by the NADPH oxidase Nox4," The Journal of Biological Chemistry, vol. 286, no. 15, pp. 1330413313, 2011.

[24] E. Rhee, L. Santiago, E. Park, P. Lad, and G. C. Bellman, "Urinary IL-6 is elevated in patients with urolithiasis," The Journal of Urology, vol. 160, no. 6, part 1, pp. 2284-2288, 1998.

[25] M. Y. Huang, L. S. Chaturvedi, S. Koul, and H. K. Koul, "Oxalate stimulates IL- 6 production in HK-2 cells, a line of human renal proximal tubular epithelial cells," Kidney International, vol. 68, no. 2, pp. 497-503, 2005.

[26] A. C. Bulua, A. Simon, R. Maddipati et al., "Mitochondrial reactive oxygen species promote production of proinflammatory cytokines and are elevated in TNFR1-associated periodic syndrome (TRAPS)," The Journal of Experimental Medicine, vol. 208, no. 3, pp. 519-533, 2011.

[27] K. Nakahira, J. A. Haspel, V. A. K. Rathinam et al., "Autophagy proteins regulate innate immune responses by inhibiting the release of mitochondrial DNA mediated by the NALP3 inflammasome," Nature Immunology, vol. 12, no. 3, pp. 222-230, 2011.

[28] R. Zhou, A. S. Yazdi, P. Menu, and J. Tschopp, "A role for mitochondria in NLRP3 inflammasome activation," Nature, vol. 469, no. 7329, pp. 221-225, 2011.

[29] S. R. Khan, S. Joshi, W. Wang, and A. B. Peck, "Regulation of macromolecular modulators of urinary stone formation by reactive oxygen species: transcriptional study in an animal model of hyperoxaluria," American Journal of PhysiologyRenal Physiology, vol. 306, no. 11, pp. F1285-F1295, 2014.

[30] Z. Jia, S. Wang, J. Tang et al., "Does crystal deposition in genetic hypercalciuric rat kidney tissue share similarities with bone formation?" Urology, vol. 83, no. 2, pp. 509.e7-509.e14, 2014.

[31] K. Tozawa, T. Yasui, A. Okada et al., "NF- $\kappa$ B activation in renal tubular epithelial cells by oxalate stimulation," International Journal of Urology, vol. 15, no. 10, pp. 924-928, 2008.
[32] Q. Xi, J. Ouyang, J. Pu, J. Hou, and S. Wang, "High concentration of calcium stimulates calcium oxalate crystal attachment to rat tubular epithelial NRK cells through osteopontin," Urology, vol. 86, no. 4, pp. 844.e1-844.e5, 2015.

[33] T. Hu, R. Luan, H. Zhang et al., "Hydrogen peroxide enhances osteopontin expression and matrix metalloproteinase activity in aortic vascular smooth muscle cells," Clinical and Experimental Pharmacology \& Physiology, vol. 36, no. 7, pp. 626-630, 2009. 


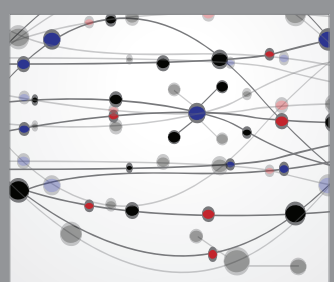

The Scientific World Journal
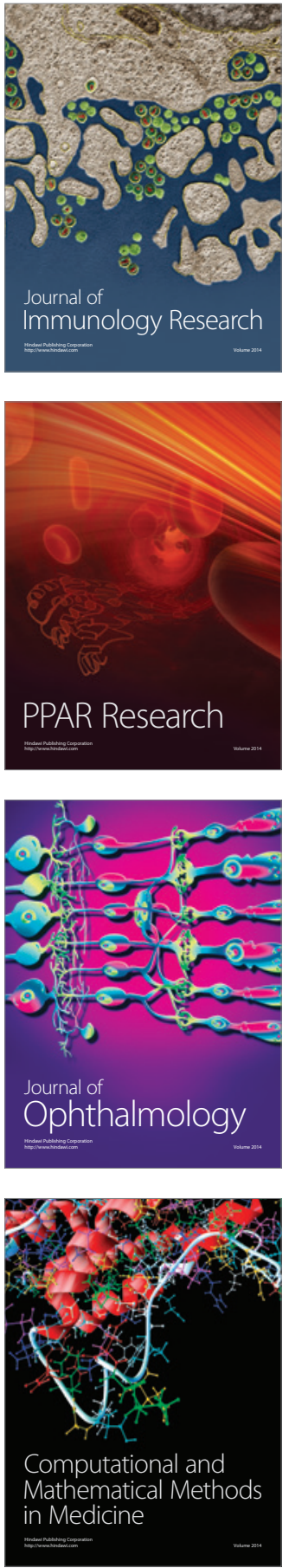

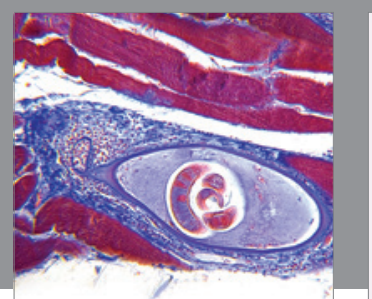

Gastroenterology Research and Practice
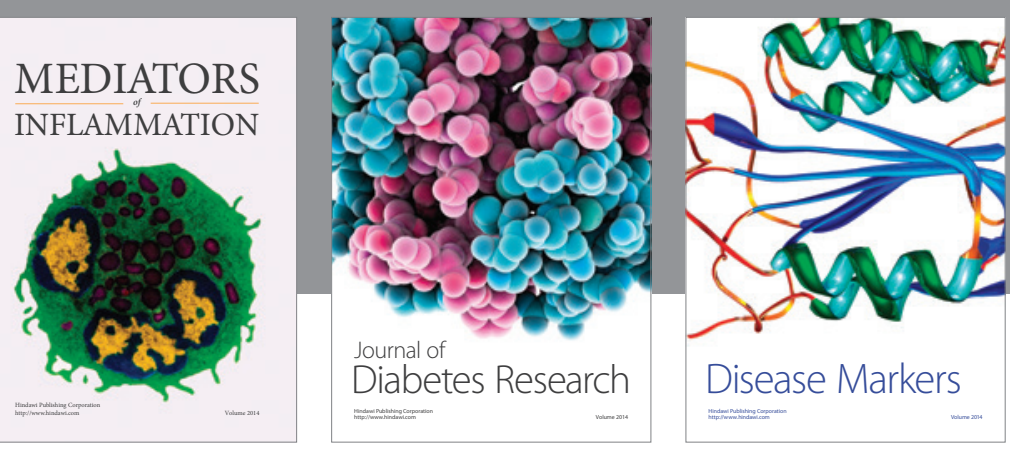

Disease Markers

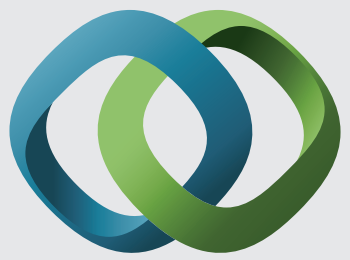

\section{Hindawi}

Submit your manuscripts at

https://www.hindawi.com
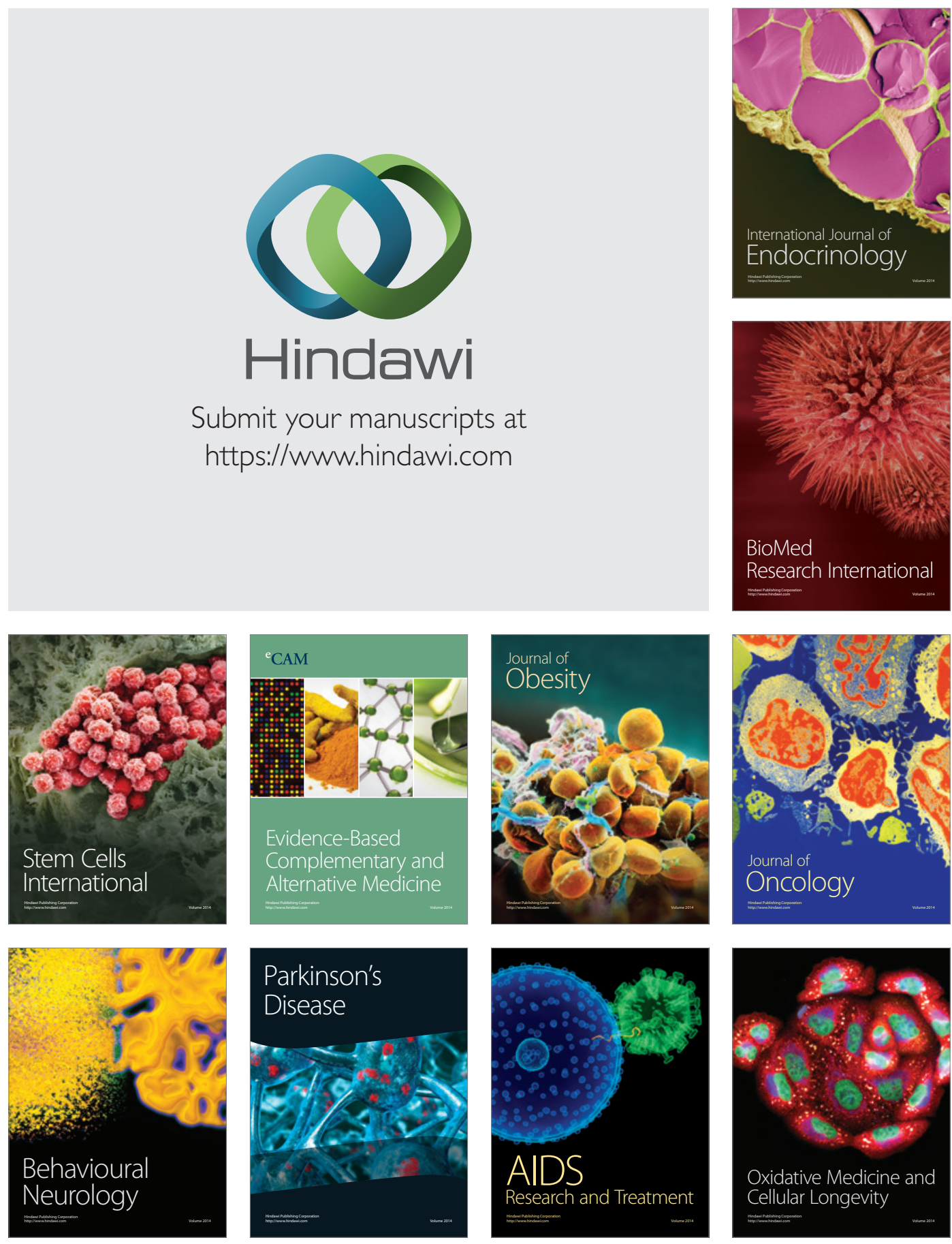